\title{
GINS2 regulates cell proliferation and apoptosis in human epithelial ovarian cancer
}

\author{
TING YAN $^{1,2}$, WENTONG LIANG $^{2}$, ENLI JIANG $^{2}$, AIZHU YE $^{3}$, QIAN WU $^{4}$ and MINGRONG XI ${ }^{1}$ \\ ${ }^{1}$ Department of Gynecology and Obstetrics, West China Second University Hospital, Sichuan University, Chengdu, \\ Sichuan 610041; Departments of ${ }^{2}$ Gynecology, ${ }^{3}$ Clinical Laboratory and ${ }^{4}$ Pathology, \\ Guizhou Provincial People's Hospital, Guiyang, Guizhou 550002, P.R. China
}

Received August 28, 2017; Accepted May 24, 2018

DOI: $10.3892 / 01.2018 .8944$

\begin{abstract}
Go-Ichi-Ni-San 2 (GINS2), also known as partner of Sld five 2, is involved in the initiation of DNA replication and cell cycle progression. GINS2 is abundantly expressed in a number of malignant solid tumors, including breast cancer, melanoma and hepatic carcinoma. However, the functions of GINS2 in epithelial ovarian cancer (EOC) remain unclear. The aim of the present study was to investigate these functions. GINS2 expression was detected in EOC and normal ovarian tissues using immunohistochemistry. To investigate the functions of GINS2 in EOC, GINS2 expression was stably knocked down in SKOV-3 cells using lentiviral short hairpin RNA (shRNA). The expression of GINS2 mRNA and protein in SKOV-3 cells was examined using reverse-transcription quantitative polymerase chain reaction (RT-qPCR) and western blot analyses, respectively. Cell proliferation was determined using high-content screening and MTT assays. Cell cycle progression and apoptosis were detected using flow cytometry. Compared with normal ovarian tissues, EOC tissues expressed increased levels of GINS2 expression (16.7 vs. 58.3\%). Increased expression of GINS2 mRNA was also observed in SKOV-3 and OVCAR3 cells. In the investigation of GINS2 functions in EOC, GINS2 expression at the mRNA and protein levels was significantly inhibited by specific GINS2 shRNA. GINS2 knockdown significantly inhibited the proliferation and viability of SKOV-3 cells and induced cell
\end{abstract}

Correspondence to: Dr Mingrong Xi, Department of Gynecology and Obstetrics, West China Second University Hospital, Sichuan University, 20 Section 3 Renming South Road, Chengdu, Sichuan 610041, P.R. China

E-mail: xmrjzz@126.com

Abbreviations: EOC, epithelial ovarian cancer; GINS, Go-Ichi-Ni-San; FA, Fanconi's anemia; TNBC, triple negative breast cancer; CBTCCCAS, Cell Bank Type Culture Collection of Chinese Academy of Sciences; FBS, fetal bovine serum; RT-qPCR, reverse transcription-quantitative polymerase chain reaction; PI, propidium iodide; CDK, cyclin-dependent kinase

Key words: Go-Ichi-Ni-San 2, expression, proliferation, apoptosis, SKOV-3 cycle arrest in S phase. Furthermore, GINS2 knockdown in SKOV-3 cells significantly increased cell apoptosis. GINS2 is markedly expressed in EOC tissues and cell lines. Stable GINS2 knockdown in SKOV-3 cells significantly inhibited cell proliferation and induced cell cycle arrest and cell apoptosis. Therefore, GINS2 may be involved in EOC progression.

\section{Introduction}

Ovarian cancer (OC) is a typically fatal disease worldwide, with $>225,000$ novel cases and >140,000 mortalities annually $(1,2)$. Symptoms associated with OC are non-specific, therefore the majority of patients with OC are diagnosed at advanced stages of the disease, which markedly increases the difficulty of treatment and decreases the survival time (3). Owing to late diagnosis and poor response to treatment, $\mathrm{OC}$ is ranked as the eighth most common cause of cancer-associated mortality in women worldwide (4). The origin of epithelial ovarian cancer (EOC) is the epithelial cells of the ovary, and EOC accounts for $\sim 90 \%$ of all ovarian cancers (5). Extensive effort has been made to investigate the pathogenesis of EOC. It has been demonstrated that the pathogenesis of EOC is associated with multiple gene mutations (e.g., in genes encoding AT-rich-interacting domain-containing protein 1A or breast cancer 1/2) (6,7), gene overexpression (e.g., in genes encoding cyclin D1 or human epidermal growth factor receptor 2) $(8,9)$ and dysregulation of signaling pathways (e.g., phosphoinositide 3 -kinase/protein kinase B or Wnt/ $\beta$-catenin) $(10,11)$. However, limited progress has been achieved in improving the prognosis and treatment of this disease. Therefore, comprehensive investigation of the pathogenesis of EOC is required.

Go-Ichi-Ni-San (GINS, meaning five, one, two and three in Japanese) complexes, first identified by Takayama et al (12), consist of partner of Sld five (PSF)1, PSF2, PSF3 and SLD5 (12). GINS complexes are a type of nucleic acid replication factor and initiate a cyclic structure that serves a significant function in the initiation of DNA replication (13). GINS2, also known as PSF2, is encoded by the GINS2 gene located in humans at chromosomal locus 16q24 (14). It has been demonstrated that GINS2 is the central component of the CMG [cell division cycle 45 (Cdc45)-minichromosome maintenance (MCM)-GINS] complex, and GINS2 is involved in the initiation of DNA replication and cell cycle progression (15). 
Tumini et al (16) identified a novel crosstalk between DNA replication and the Fanconi's anemia (FA) signaling pathway, in which GINS and the core complex help to load or stabilize the FA core complex onto chromatin, and GINS2 depletion is insufficient to decrease the monoubiquitylation of FA complementation group D2 or its localization to nuclear foci following DNA damage (16).

A previous study identified that GINS2 is associated with the occurrence of genomic DNA damage in untransformed human fibroblasts (17), suggesting that GINS2 may be involved in the process of tumorigenesis. A gene expression meta-analysis identified GINS2 at 16q24 as a potential metastasis-promoting genes in breast cancer (18). Further studies demonstrated that increased GINS2 expression was associated with advanced stage of tumor, poor relapse-free survival, poor distant metastasis-free survival and poor tamoxifen efficacy in patients with breast cancer $(19,20)$. An in vitro study identified that GINS2 expression was enriched in triple negative breast cancer (TNBC) cell lines, and GINS2 silencing decreased cell proliferation, invasive capability and stem-like properties of TNBC cells (21). Therefore, GINS2 has been considered as a potential prognostic marker and therapeutic target in breast cancer. On the basis of the analysis of genome-wide gene expression profiles, GINS2 has been identified as a tumor-node-metastasis stage-associated gene in lung adenocarcinoma (22). Furthermore, GINS2 serves important functions in regulating cell proliferation, apoptosis and cell cycle transition in leukemic cell lines $(23,24)$. However, to the best of our knowledge, the functions of GINS2 in EOC have not been investigated.

In the present study, the expression of GINS2 was investigated in EOC and normal ovarian tissues using immunohistochemistry and the effects of GINS2 on cell proliferation (using cell counting and MTT assays), cell cycle transition (using propidium iodide staining) and cell apoptosis [using Annexin V-allophycocyanin (APC) staining] were further studied in an EOC cell line, SKOV-3. The results of the present study provide evidence for the potential functions of GINS2 in EOC.

\section{Materials and methods}

Cell line culture. The human EOC cell line SKOV-3 was purchased from the Cell Bank Type Culture Collection of Chinese Academy of Sciences (CBTCCCAS; Shanghai, China) and another EOC cell line, OVCAR3, was purchased from the American Type Culture Collection (Manassas, VA, USA). SKOV-3 cells were cultured in McCoy's 5A medium (Invitrogen; Thermo Fisher Scientific, Waltham, MA, USA) with $10 \%$ fetal bovine serum (FBS; Ausbian, Sydney, Australia), and OVCAR3 cells were maintained in RPMI-1640 medium (Corning Incorporated, Corning, NY, USA) with $20 \%$ FBS. 293T cells were obtained from the CBTCCCAS and were cultured in Dulbecco's modified Eagle's medium (DMEM; Corning Incorporated) with $10 \%$ FBS. All cells were cultured in a humidified atmosphere at $37^{\circ} \mathrm{C}$ with $5 \% \mathrm{CO}_{2}$.

Establishment of stable GINS2 knockdown in SKOV-3 cells. For stable knockdown of GINS2, GINS2 (target sequence, GATTAACCTGAAACAAAGA) or negative control (target sequence, TTCTCCGAACGTGTCACGT) short hairpin
RNAs (shRNAs) were cloned into lentiviral vector GV115-GFP (Shanghai GeneChem Co., Ltd., Shanghai, China). Lentiviral plasmids were purified and transfected together with pHelper 1.0 and pHelper 2.0 plasmids (Shanghai GeneChem Co., Ltd.) into 293 T cells (in 10 -cm plates) using Lipofectamine ${ }^{\circledR} 2000$ (Invitrogen; Thermo Fisher Scientific, Inc.). Culture medium containing lentiviruses was collected 48 and $72 \mathrm{~h}$ after transfection and was used to infect SKOV-3 cells in the presence of $5 \mu \mathrm{g} / \mathrm{ml}$ Polybrene (Invitrogen; Thermo Fisher Scientific, Inc.) for $12 \mathrm{~h}$. At 3 days after infection, green fluorescent protein (GFP) expression was observed under magnification, x100 using phase contrast fluorescent microscopy (Olympus Corporation, Tokyo, Japan).

Reverse transcription-quantitative polymerase chain reaction $(R T-q P C R)$. Total RNA from SKOV-3 and OVCAR3 cells was extracted using TRIzol ${ }^{\circledR}$ reagent (Thermo Fisher Scientific, Inc.) according to the manufacturer's protocol. A $2-\mu \mathrm{g}$ amount of total RNA was used to synthesize first-strand DNA using Moloney murine leukemia virus reverse transcriptase (Promega Corporation, Madison, MI, USA) and oligo(dT) primers (Sangon Biotech Co., Ltd., Shanghai, China). GINS2 mRNA expression was determined using qPCR with SYBR master mixture (Takara Biotechnology Co., Ltd., Dalian, China) on a LightCycler 480 instrument (Roche Diagnostics, Basel, Switzerland). qPCR was carried out at $95^{\circ} \mathrm{C}$ for $30 \mathrm{sec}$, then 40 cycles of $95^{\circ} \mathrm{C}$ for $5 \mathrm{sec}$ and $60^{\circ} \mathrm{C}$ for $30 \mathrm{sec}$. GAPDH was used as an internal control for quantification. The primers for PCR analysis were as follows: GINS2 forward, 5'-CAG AAATGTCGCCTGCTCC-3' and reverse, 5'-GGATTTCGT CTGCCTTCG-3'; GAPDH forward, 5'-TGACTTCAACAG CGACACCCA-3' and reverse, 5'-CACCCTGTTGCTGTA GCCAAA-3'. Relative gene expression was calculated using the $2^{-\Delta \Delta \mathrm{Cq}}$ method and normalized to GAPDH expression, as described previously (25).

Western blot analysis. Cells at between 36 and $48 \mathrm{~h}$ after viral infection were lysed in lysis buffer containing $100 \mathrm{mM}$ Tris/ $\mathrm{HCl}$ (pH 6.8), 4\% SDS, 20\% glycerol and 2\% mercaptoethanol. Protein concentration was assessed using a bicinchoninic acid protein assay kit (Beyotime Institute of Biotechnology, Haimen, China), according to the manufacturer's protocol. An equal amount of total protein $(20 \mu \mathrm{g})$ from each sample was resolved by SDS-PAGE (10\% gels) and transferred onto polyvinylidene fluoride membranes. The membranes were blocked in 5\% non-fat milk solution in Tris-buffered saline and Tween-20 (TBST) at room temperature for $1 \mathrm{~h}$ and then further incubated with the primary antibody for $2 \mathrm{~h}$. The primary antibodies used were mouse anti-GINS2 (cat. no. SAB1409430; 1:2,000 dilution; Sigma-Aldrich; Merck KGaA, Darmstadt, Germany) and mouse anti-GADPH (cat. no. sc-47724; 1:2,000 dilution; Santa Cruz Biotechnology, Inc., Dallas, TX, USA). Next, the membranes were washed with TBST three times and incubated with horseradish peroxidase-conjugated secondary goat anti-mouse IgG (cat. no. sc-2005, 1:2,000 dilution; Santa Cruz Biotechnology, Inc.) for $2 \mathrm{~h}$. The signals were detected using enhanced chemiluminescence reagent (Thermo Fisher Scientific, Inc.).

Cell proliferation assays. Following infection with lentiviruses expressing GINS2 or scrambled shRNA, the proliferation of 

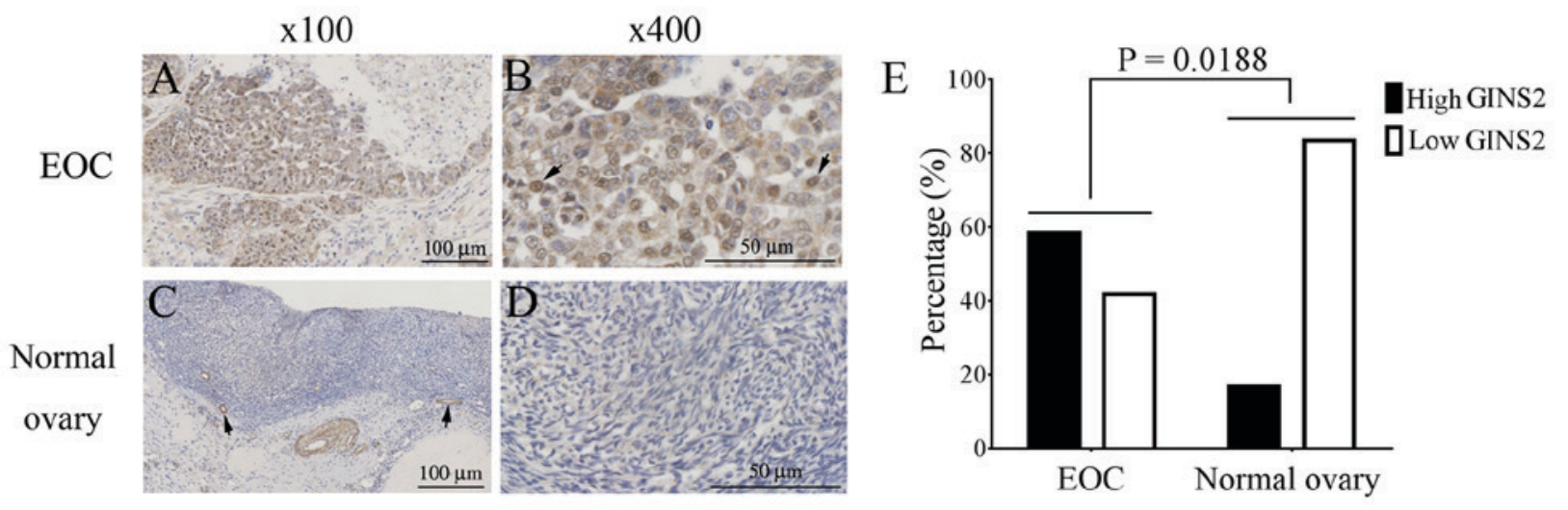

Figure 1. Expression of GINS2 in ovarian cancer and normal ovarian tissues. Representative images of GINS2 staining in EOC tissues at (A) x100 and (B) x400 magnification, and in normal ovarian tissues at (C) x100 and (D) x400 magnification. Arrows in (B) and (C) indicate nuclei staining and blood vessel staining, respectively. (E) Quantification of GINS2 expression in EOC tissues and normal ovarian tissues, which was determined by the staining intensity and the percentage of positive cells. The threshold values for high or low GINS2 expression were $>6$ or $\leq 6$, respectively. The P-value was calculated by two-tailed Fisher's exact test. GINS, Go-Ichi-Ni-San; EOC, epithelial ovarian cancer.

SKOV-3 cells expressing GINS2 shRNA or scrambled shRNA was determined using high-content screening. SKOV-3 cells were cultured for $48 \mathrm{~h}$ and then split at the exponential growth phase into 96 -well plates in triplicate at a density of 2,000 cells/well. Cells were cultured for 5 days, and cell images were captured once daily using Celigo (Nexcelom, Lawrence, MA, USA). The cell numbers in each well were determined at different time points using Celigo software (version 2.1), and cell proliferation curves were generated.

Cell proliferation was also assessed using an MTT assay. Stable SKOV-3 cells expressing SKOV-3 or scrambled shRNA were seeded in 96-well plates at a density of 2,000 cells/well and incubated at $37^{\circ} \mathrm{C}$ for $1,2,3,4$ or 5 days. Then cells were incubated with MTT solution $(5 \mathrm{mg} / \mathrm{ml}$ ) for $4 \mathrm{~h}$. Following incubation, culture medium in each well was removed and $100 \mu$ ldimethyl sulfoxide was added to solubilize the formazan salt. After $5 \mathrm{~min}$, the optical density at $490 \mathrm{~nm}$ was determined using a microplate reader (Tecan Infinite M2009PR; Tecan Group, Ltd., Männedorf, Switzerland).

Cell apoptosis assay. Cell apoptosis was determined using an Annexin V-APC kit (eBioscience; Thermo Fisher Scientific, Inc.), according to the manufacturer's protocol, and a Guava ${ }^{\circledR}$ easyCyte HT flow cytometer (EMD Millipore, Billerica, MA, USA). For analysis of apoptosis, the cells $\left(8.0 \times 10^{5}\right)$ were stained with $200 \mu \mathrm{l}$ binding buffer containing $10 \mu \mathrm{l}$ Annexin V-APC at room temperature in the dark for between 10 and $15 \mathrm{~min}$. Following several washes, cells were analyzed at as wavelength of $633 \mathrm{~nm}$. All experiments were performed in triplicate.

Cell cycle assay. SKOV-3 cells expressing GINS2 or scrambled shRNA were seeded into $6-\mathrm{cm}$ dishes for further culture. Upon reaching $\sim 80 \%$ confluence, $>1 \times 10^{6}$ cells were collected and fixed with ice-cold $75 \%$ alcohol for $>1 \mathrm{~h}$. Cells were then washed with D-Hanks buffer (Invitrogen; Thermo Fisher Scientific, Inc.) and stained with propidium iodide (PI) buffer $(50 \mu \mathrm{g} / \mathrm{ml}$ PI and $100 \mu \mathrm{g} / \mathrm{ml}$ RNase in D-Hanks buffer) for $30 \mathrm{~min}$ at room temperature in the dark. Following several washes, cell cycle analysis was performed for 10,000 cells using flow cytometry using a Guava easyCyte HT instrument.

Immunohistochemistry. Human ovarian tissue arrays including formalin-fixed normal and EOC tissues were purchased from Cybrdi, Inc. (Frederick, MD, USA). The tissue array (OV243; 2-mm diameter) included 36 cases of EOC samples and 12 cases of normal ovarian tissues. According to the grading criteria of The International Federation of Gynecology and Obstetrics, EOC samples consisted of 6 grade II samples, 16 grade III samples and 14 grade IV samples. None of the patients with EOC had received any treatment prior to sample collection. Following deparaffinization and rehydration, each slide was boiled in $10 \mathrm{mmol} / 1$ citrate buffer ( $\mathrm{pH}$ 6.0) for $20 \mathrm{~min}$ and then blocked with $10 \%$ bovine serum albumin in Tris-buffered saline for $30 \mathrm{~min}$. Next, the slides were incubated with the primary anti-GINS2 antibody (cat. no. HPA057285; 1:500 dilution; Sigma-Aldrich; Merck $\mathrm{KGaA}$ ) at $4^{\circ} \mathrm{C}$ overnight, followed by incubation with biotinylated secondary antibody (cat. no. sc-2039; 1:2,000 dilution) for $1 \mathrm{~h}$ and streptavidin-horseradish peroxidase conjugate (cat. no. sc-52234; 1:2,000 dilution; both Santa Cruz Biotechnology, Inc.) for $30 \mathrm{~min}$. Finally, the slides were stained with diaminobenzidine (cat. no. sc-24982) for $5 \mathrm{~min}$ and then counterstained with hematoxylin (cat. no. sc-396328; both Santa Cruz Biotechnology, Inc.) for $1 \mathrm{~min}$ at room temperature. The slides were scored by two pathologists who were blinded to the clinical information of the samples. In the case of a discrepancy, the two pathologists reviewed the slides simultaneously to achieve a consensus. GINS2 scoring was performed by evaluating the intensity of staining and the extent of staining in the whole tissue. For intensity, 0 indicated negative staining, 1 indicated weak staining, 2 indicated moderate staining and 3 indicated strong staining. For the extent of staining area, 0 indicated $0 \%$ staining, 1 indicated 1-25\% staining, 2 indicated 26-50\% staining, 3 indicated $51-75 \%$ staining, and 4 indicated $76-100 \%$ staining. The final immunoreactive score was determined by multiplying the intensity score by the extent score, with the minimum score attainable being 0 and a maximum score being 12 . Scores $\leq 6$ were considered as low GINS2 
A
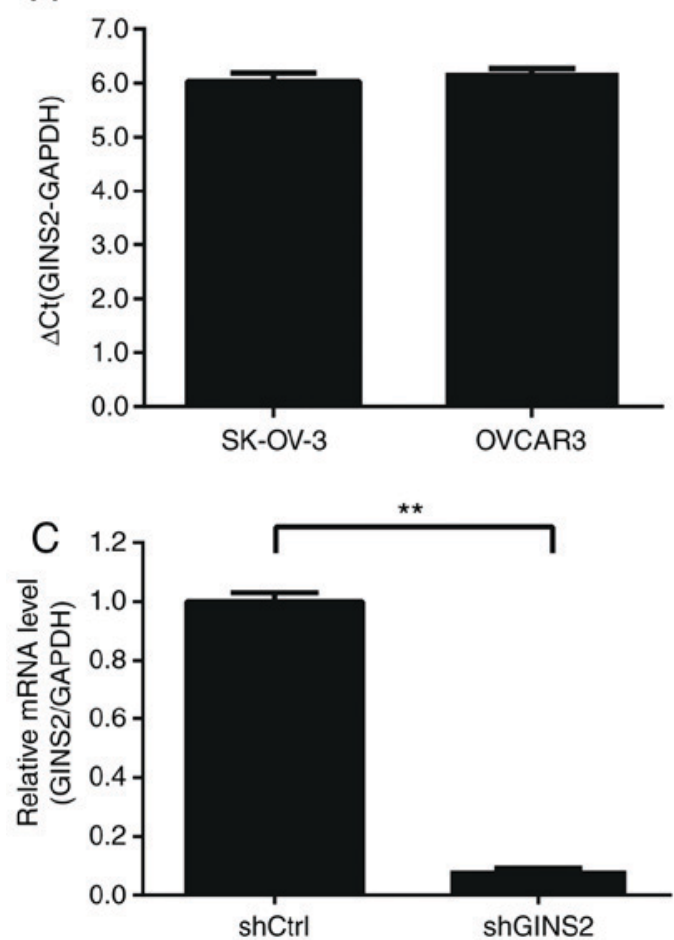

B
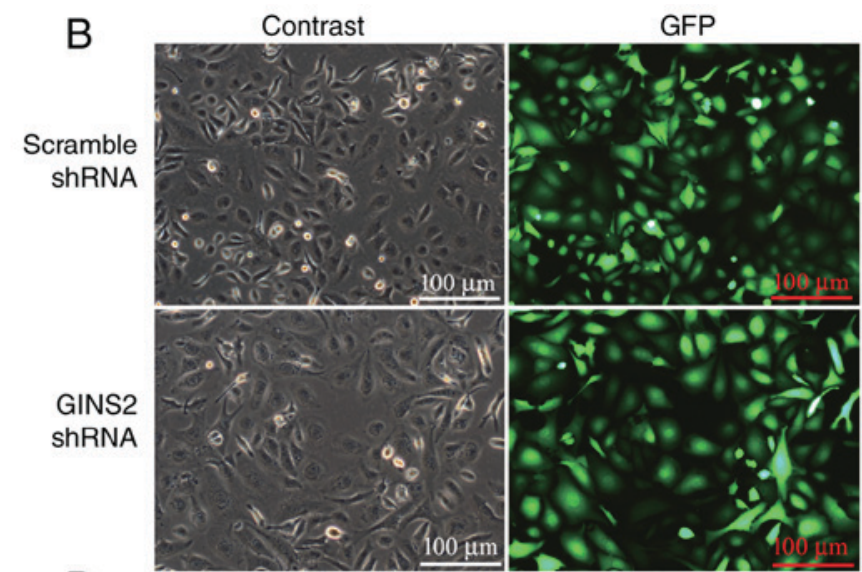

$\mathrm{D}$

GINS2

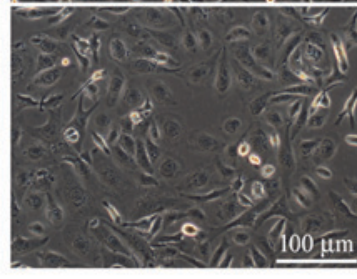

shGINS2

GINS2

shCtrl

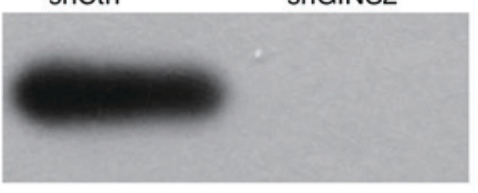

GAPDH

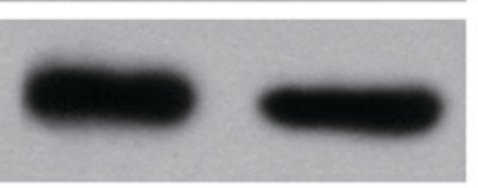

Figure 2. Stable GINS2 knockdown in SKOV-3 cells. (A) Expression of GINS2 mRNA in SKOV-3 and OVCAR3 cell lines, determined using the reverse transcription-quantitative polymerase chain reaction. (B) Infection efficiency of lentivirus expressing GFP and scrambled or GINS shRNA. At $48 \mathrm{~h}$ after infection, GFP signals were captured by fluorescence microscopy, and the phase-contrast images of cells are presented as a comparison. (C) Effect of specific GINS2 shRNA on GINS2 mRNA expression in SKOV-3 cells at 5 days after infection. GAPDH was used as an internal control. The difference was analyzed by one-way analysis of variance. ${ }^{* *} \mathrm{P}<0.01$ from three $(\mathrm{n}=3)$ independent experiments. (D) Effect of specific GINS2 shRNA on GINS2 protein expression in SKOV-3 cells. GAPDH was used as an internal control. GINS, Go-Ichi-Ni-San; GFP, green fluorescent protein; shRNA/sh, short hairpin RNA; Ctrl, control.

expression, whereas scores $>6$ were considered as high GINS2 expression.

Statistical analysis. Statistical analysis was performed using SPSS software (version 17.0; SPSS, Inc., Chicago, IL, USA). All results are expressed as the mean \pm standard deviation of multiple experiments. Results were analyzed by one-way analysis of variance and two-tailed Fisher's exact test. $\mathrm{P}<0.05$ was considered to indicate a statistically significant difference.

\section{Results}

GINS2 is overexpressed in EOC tissues, but not in normal ovarian tissue. Since GINS2 was highly expressed in breast cancer and lung adenocarcinoma $(19,22)$, GINS2 expression was determined in 36 samples of EOC tissues and 12 samples of normal ovarian tissues using immunohistochemical staining. Strong GINS2 signals were identified in EOC tissues, primarily in the nuclei of carcinoma cells (Fig. 1A and B), whereas GINS2 expression was weak in normal ovarian tissue, with some signals in blood vessels (Fig. 1C and D). GINS2 staining was quantified on the basis of intensity and percentage of GINS2-positive cells. GINS2 was highly expressed in 21/36 EOC tissue samples (58.33\%), but in only 2/12 normal ovarian tissue samples $(16.67 \%)$. The difference in GINS2 expression between EOC and normal ovarian tissues was statistically significant $(\mathrm{P}=0.0188$; Fig. 1E).
Stable knockdown of GINS2 expression in EOC cells. To investigate the functions of GINS2 in EOC cells, the expression of GINS2 mRNA was determined in the EOC cell lines SKOV-3 and OVCAR3 using RT-qPCR. GINS2 mRNA was highly expressed in these two cell lines (Fig. 2A). The SKOV-3 cell line was selected for further functional analyses.

GINS2 mRNA expression was stably knocked down in SKOV-3 cells using a specific shRNA in a lentiviral vector. Lentiviruses expressing GINS2 shRNA or scrambled shRNA were produced by $293 \mathrm{~T}$ cells and were used to infect SKOV-3 cells. At $48 \mathrm{~h}$ after infection, it was observed that $>80 \%$ of SKOV-3 cells were GFP-positive, indicating a high infection efficiency (Fig. 2B). At 5 days after infection, the mRNA and protein expression levels of GINS2 in SKOV-3 cells were determined using RT-qPCR and Western blotting, respectively. The mRNA expression level of GINS2 was significantly inhibited ( $>90 \%$ knockdown) by specific GINS2 shRNA compared with scrambled shRNA ( $\mathrm{P}<0.001$; Fig. $2 \mathrm{C})$ and GINS2 protein expression was identified to be markedly decreased following GINS2 knockdown by shRNA (Fig. 2D).

GINS2 knockdown inhibits SKOV-3 cell proliferation. To investigate the function of GINS2 in cell proliferation, the proliferation of SKOV-3 cells expressing GINS2 shRNA or scrambled shRNA was determined using high-content screening. As presented in Fig. 3A, SKOV-3 cell proliferation was significantly inhibited by GINS2 shRNA $(\mathrm{P}<0.001)$. Compared with the number of cells on day 1 , the number of 

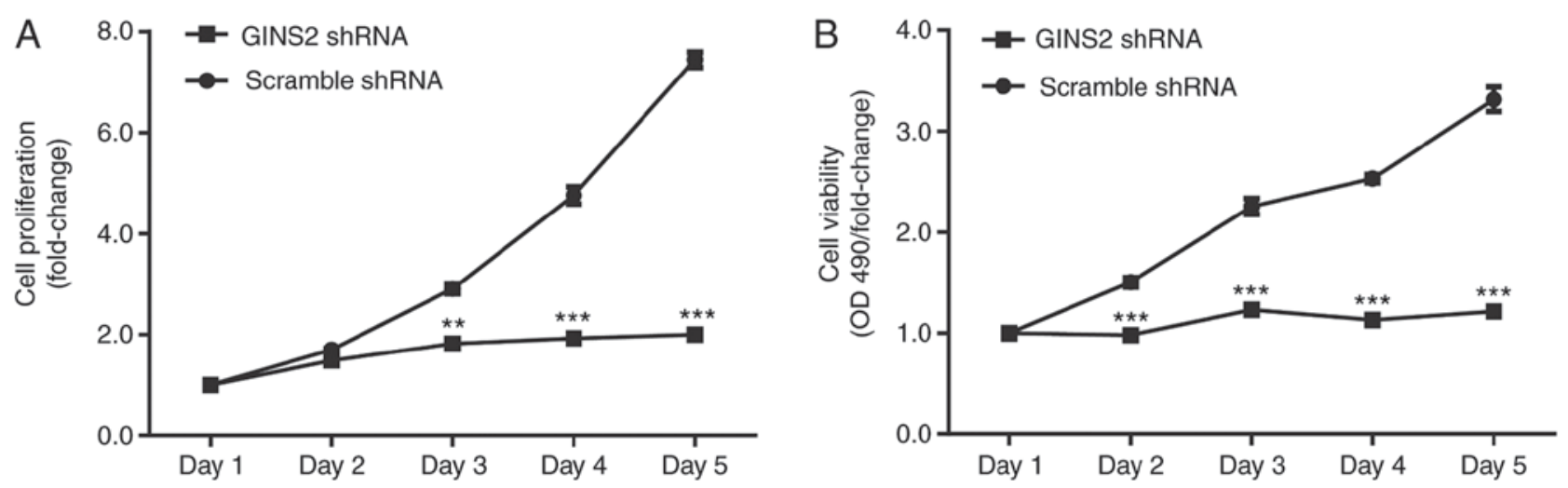

Figure 3. GINS2 knockdown inhibits cell proliferation in SKOV-3 cells. (A) Effect of scrambled shRNA and GINS2 shRNA on cell proliferation, which was monitored by high-content screening. Cell numbers at different time points were determined using Celigo software. The cell number on day 1 in each group was set as 1, and the cell number at the other time points was compared with day 1. (B) Effect of scrambled shRNA and GINS2 shRNA on cell viability, investigated using an MTT assay. Cell viability was assessed by determining the $\mathrm{OD}_{490}$ value. The $\mathrm{OD}_{490}$ value on day 1 was set as 1 , and the $\mathrm{OD}_{490}$ values at the other time points were compared with day 1 . The differences in cell number and viability between the two groups at different time points were analyzed by one-way analysis of variance. ${ }^{* * *} \mathrm{P}<0.01$ and ${ }^{* * * *} \mathrm{P}<0.001$ from three $(\mathrm{n}=3)$ independent experiments. GINS, Go-Ichi-Ni-San; shRNA, short hairpin RNA; OD, optical density.

cells in the scrambled shRNA group increased $7.45 \pm 0.15$-fold after 5 days, whereas it increased only $2.00 \pm 0.10$-fold in the GINS2 group (Fig. 3A).

The effect of GINS2 knockdown on SKOV-3 cell viability was investigated using an MTT assay. Compared with day 1 , cell viability in the scrambled shRNA group gradually increased between day 1 and day 5 (a 3.316 \pm 0.122 -fold increase by day 5), whereas cell viability increased only slightly between day 1 and day 5 (increased by $1.215 \pm 0.0324$ fold on day 5). The difference in cell viability between the two groups was statistically significant after 2 and 3 days $(\mathrm{P}<0.01$ and $\mathrm{P}<0.001$; Fig. 3B).

GINS2 knockdown induces cell cycle arrest in SKOV-3 cells. Since GINS2 knockdown inhibited SKOV-3 cell proliferation, it was investigated whether GINS2 knockdown affects SKOV-3 cell cycle progression. Cell cycle analysis was performed using PI staining and assessed using flow cytometry. Results of cell cycle progression are presented in Fig. 4A. Cell cycle analysis revealed that, compared with scrambled shRNA, GINS2 shRNA significantly decreased the proportion of cells in the $\mathrm{G}_{0} / \mathrm{G}_{1}$ phase $(45.25 \pm 0.23$ compared with $42.79 \pm 1.46 \%$; $\mathrm{P}<0.05)$ and $\mathrm{G}_{2} / \mathrm{M}$ phase $(19.13 \pm 0.49$ compared with $16.33 \pm 0.23 \%$; $\mathrm{P}<0.01$ ), whereas the percentage of cells in $\mathrm{S}$ phase was significantly increased $(35.62 \pm 0.29$ compared with $40.88 \pm 1.25 \%$; $\mathrm{P}<0.01$ ) following GINS2 knockdown (Fig. 4B).

GINS2 knockdown induces apoptosis in SKOV-3 cells. It was investigated whether GINS2 knockdown affects apoptosis using Annexin V-APC staining and flow cytometry. Representative apoptosis assay results are presented in Fig. 5A. Compared with scrambled shRNA, GINS2 shRNA significantly increased apoptosis $(4.57 \pm 0.40$ compared with $20.97 \pm 1.63 \%$; P $<0.01$; Fig. 5B).

\section{Discussion}

GINS, a ring-like protein complex involving PSF1, PSF2 (GINS2), PSF3 and SLD5, was initially extracted from budding yeast (12). GINS2, as an important subunit of GINS complexes, mediates the interaction between MCM complexes and $\mathrm{Cdc} 45$ (CDC45-MCM-2-7-GINS) at the initiation of DNA replication in eukaryotic cells (15). GINS2 has been associated with the malignancy of a number of types of cancer (20-24). GINS2 is highly expressed in many malignant tumors, including breast cancer and acute promyelocytic leukemia $(20,21,23)$. Furthermore, high GINS2 expression is associated with advanced stage of tumor, poor relapse-free survival times, poor distant metastasis-free survival times and poor tamoxifen efficacy in patients with breast cancer (20). Therefore, GINS2 has been considered as a potential prognostic marker and therapeutic target in breast cancer. However, to the best of our knowledge, the association between GINS2 expression and the progression of EOC remains to be clarified. In the present study, high expression of GINS2 was observed in EOC tissues and cell lines. Furthermore, stable GINS2 knockdown in SKOV-3 cells was able to significantly inhibit cell proliferation, and induce cell cycle arrest and apoptosis.

It has been demonstrated that the GINS2 transcript is highly expressed in breast cancer samples, and its level is associated with lung metastasis, histological grade, and acquired endocrine therapy resistance in patients with breast cancer (20). A study by Liu et al (22) identified GINS2 as a differentially expressed gene (high expression) in 90 lung adenocarcinoma samples using gene microarray analysis. In the present study, GINS2 was identified, for the first time, to be expressed at high levels in EOC samples from 36 patients and in two human EOC cell lines. In patients with breast cancer and lung adenocarcinoma, GINS2 expression was positively associated with the histological grade of tumors, in which high expression of GINS2 was observed in $17.2 \%$ of grade $1,50 \%$ of grade 2 and $77.1 \%$ of grade 3 breast cancer samples, and in stage II lung adenocarcinomas $(20,22)$. The EOC samples assessed in the present study were purchased from a commercial company. Since the number of patients with EOC was limited and data for the clinicopathological parameters, including lung metastasis, progress-free survival, prognosis, therapy resistance, were not provided by the company, GINS2 protein expression was compared only 

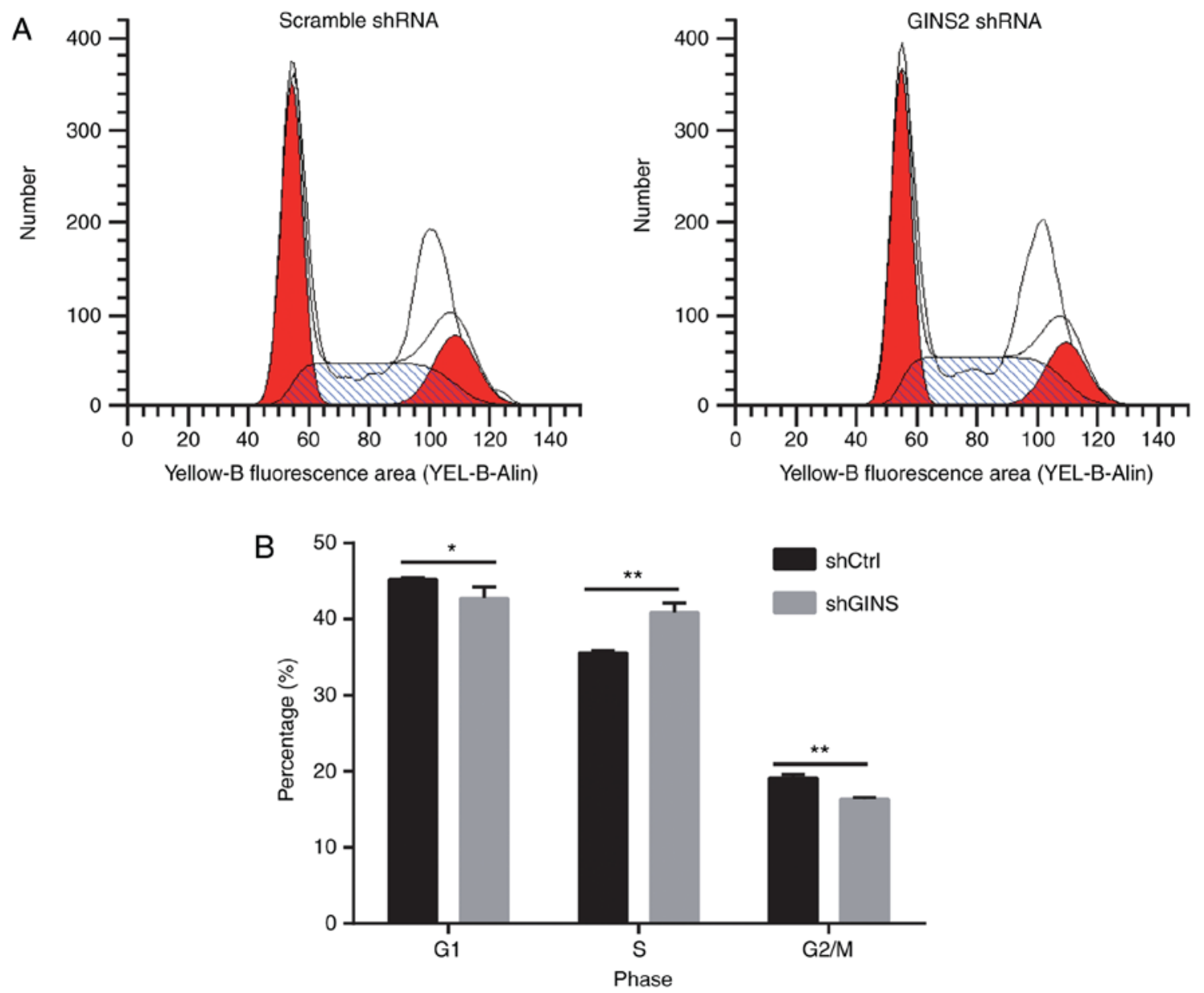

Figure 4. GINS2 knockdown induces cell cycle arrest at S phase. (A) Representative cell cycle analysis results in the two groups, as determined using propidium iodide staining. (B) Quantification of cell cycle analysis for the two groups. The differences in the proportions at different cell cycle phases between the two groups were analyzed by one-way analysis of variance. ${ }^{*} \mathrm{P}<0.05$ and ${ }^{* *} \mathrm{P}<0.01$ from three $(\mathrm{n}=3)$ independent experiments. GINS, Go-Ichi-Ni-San; shRNA/sh, short hairpin RNA; Ctrl, control.
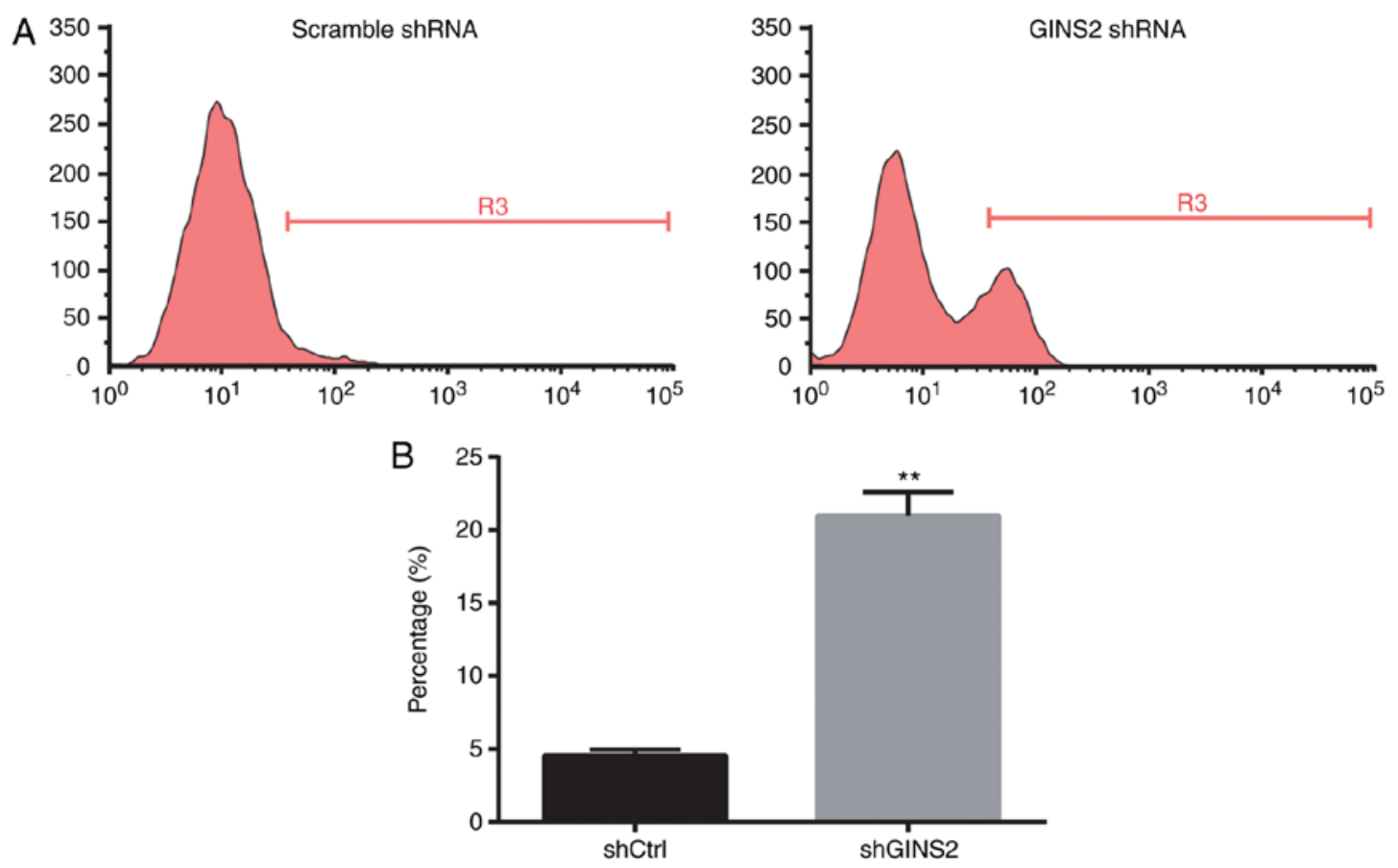

Figure 5. GINS2 knockdown induces apoptosis. (A) Representative cell apoptosis analysis in the two groups, as determined by Annexin V-allophycocyanin staining. (B) Quantification of apoptotic cells in the two groups. Differences were analyzed by one-way analysis of variance. ${ }^{* *} \mathrm{P}<0.01 \mathrm{vs}$. shCtrl, from three $(\mathrm{n}=3)$ independent experiments. GINS, Go-Ichi-Ni-San; shRNA/sh, short hairpin RNA; Ctrl, control. 
between EOC samples and normal ovarian tissues. Therefore, in future studies, GINS2 expression should be investigated in a large number of patients with EOC of different histological grades and with complete clinicopathological data in order to assess the association between GINS2 protein expression and other clinicopathological parameters of EOC.

Since GINS2 mediates the interaction between MCM complexes and $\mathrm{Cdc} 45$ at the initiation of DNA replication in eukaryotic cells (15), it is reasonable to hypothesize that GINS2 is involved in the regulation of cell cycle progression and proliferation. It has been demonstrated that GINS2 serves important functions in the proliferation of leukemia cells $(23,24)$. In leukemia cell lines (HL60, K562 and NB4), GINS2 knockdown by siRNA significantly suppressed cell proliferation and induced cell cycle arrest at the $G_{2}$ phase through activation of the $\mathrm{p} 38$ mitogen-activated protein kinase signaling pathway, whereas overexpression of GINS2 in HL60 cells promoted cell proliferation $(23,24)$. In the present study, it was also identified that GINS2 knockdown in SKOV-3 cells significantly suppressed cell proliferation. However, GINS2 knockdown induced cell cycle arrest at the $\mathrm{S}$ phase, but not the $G_{2}$ phase as demonstrated in leukemia cells. Eukaryotic cell cycle progression is tightly regulated by the complexes consisting of cyclins and cyclin-dependent kinases (CDKs), in which cyclin D-CDK4/6 is required for $\mathrm{G}_{1}$ progression, cyclin E-CDK2 is required for the $\mathrm{G}_{1}-\mathrm{S}$ transition, cyclin A-CDK2 is required for $\mathrm{S}$ phase progression and cyclin $\mathrm{A} / \mathrm{B}-\mathrm{CDC} 2$ is required for the $\mathrm{G}_{2}-\mathrm{M}$ transition $(26,27)$. Thus, the expression of cyclins and CDKs is often used to assess cell proliferation and cell cycle transition. Although the effect of GINS2 knockdown on the expression of cyclins and CDKs was not investigated in SKOV-3 cells, previous studies demonstrated that GINS2 knockdown decreases the expression of cyclin A, cyclin B1, cyclin D1 and CDK1 in leukemia cells $(23,24)$. These results support the hypothesis that GINS2 knockdown induces cell cycle arrest at the $\mathrm{S}$ or $\mathrm{G}_{2}$ phase. Furthermore, aberrant cell cycle regulation is the major cause for over-proliferation of tumor cells. The $\mathrm{S}$ phase is a critical period during which cells commit to proliferation or growth arrest (26). Individual replication origins are activated at the onset of S phase through the assembly of replication factors, including Sld3, GINS, Cut5, Drc1, Cdc45, replication protein A and the DNA polymerase $\alpha$-primase complex (28). GINS2 regulates the chromosome segregation, probably through its function in centromere replication at the S phase (29). In eukaryotes, the process of DNA replication occurs at $S$ phase in a highly coordinated manner, in which GINS associates with replication origins and then with neighboring fragments during this period (30). Understanding the regulation of the $\mathrm{S}$ phase transition is central to clarifying the pathogenesis of many diseases, particularly cancer (31).

Uncontrolled cell proliferation and decreased apoptosis are two major characteristics of the majority of cancer cells. In the present study, it was observed that GINS2 knockdown significantly induced apoptosis in SKOV-3 cells. Consistent with these results, previous studies also demonstrated that GINS2 knockdown induced apoptosis in leukemia cells $(23,24)$. Furthermore, GINS2 knockdown significantly increased the expression of the pro-apoptotic protein B-cell lymphoma 2 (Bcl-2)-associated X protein, but decreased the expression of the anti-apoptotic protein Bcl-2 (23,24), although the underlying molecular mechanisms remain to be clarified. Since the present study is, to the best of our knowledge, the first to investigate GINS2 functions in EOC, the expression pattern of GINS2 was initially investigated in EOC samples and then investigated major functions (cell proliferation and apoptosis) of GINS2 in an EOC cell line. The results of the present study provide a solid basis for future studies, in which the functions of GINS2 in EOC and precise underlying molecular mechanisms should be validated in vitro and in vivo.

In summary, the results of the present study demonstrate that GINS2 was highly expressed in human EOC samples and cell lines. The silencing of GINS 2 expression in SKOV-3 cells inhibited cell proliferation, induced cell cycle arrest at $S$ phase and increased apoptosis. Therefore, these results suggest that GINS2 may be involved in the progression and malignancy of EOC, although the functions of GINS2 in the tumorigenesis of human EOC and underlying molecular mechanisms require further investigation.

\section{Acknowledgements}

Not applicable.

\section{Funding}

The present study was supported by The Youth Fund of Guizhou Provincial People's Hospital (grant no. GZSYQN[2017]09).

\section{Availability of data and materials}

The datasets used and/or analyzed during the current study are available from the corresponding author on reasonable request.

\section{Authors' contributions}

TY and MX conceived and designed the study. TY, EJ, AY, WL and QW performed the experiments. TY and WL wrote the paper. TY, WL, EJ, AY and MX reviewed and edited the manuscript. All authors read and approved the manuscript.

\section{Ethics approval and consent to participate}

Not applicable.

\section{Consent for publication}

Not applicable.

\section{Competing interests}

The authors declare that they have no competing interests.

\section{References}

1. Ferlay J, Shin HR, Bray F, Forman D, Mathers C and Parkin DM: Estimates of worldwide burden of cancer in 2008: GLOBOCAN 2008. Int J Cancer J Int Du Cancer 127: 2893-2917, 2010.

2. Siegel R, Naishadham D and Jemal A: Cancer statistics, 2013. CA Cancer J Clin 63: 11-30, 2013.

3. Brown PO and Palmer C: The preclinical natural history of serous ovarian cancer: Defining the target for early detection. PLoS Med 6: e1000114, 2009. 
4. Torre LA, Bray F, Siegel RL, Ferlay J, Lortet-Tieulent J and Jemal A: Global cancer statistics, 2012. CA Cancer J Clin 65: 87-108, 2015.

5. Auersperg N, Wong A, Choi K, Kang S and Leung P: Ovarian surface epithelium: Biology, endocrinology, and pathology. Endocr Rev 22: 255-288, 2001.

6. Wu JN and Roberts CWM: ARID1A mutations in cancer: Another epigenetic tumor suppressor? Cancer Discov 3: 35-43, 2013.

7. Maistro S, Teixeira N, Encinas G, Katayama ML, Niewiadonski VD, Cabral LG, Ribeiro RM, Gaburo Junior N, de Gouvêa AC, Carraro DM, et al: Germline mutations in BRCA1 and BRCA2 in epithelial ovarian cancer patients in Brazil. BMC Cancer 16: 934, 2016.

8. Hashimoto T, Yanaihara N, Okamoto A, Nikaido T, Saito M, Takakura S, Yasuda M, Sasaki H, Ochiai K and Tanaka T: Cyclin D1 predicts the prognosis of advanced serous ovarian cancer. Exp Ther Med 2: 213-219, 2011.

9. Chao WR, Lee MY,Lin WL, Chen CK, Lin JC, Koo CL, Sheu GT and Han CP: HER2 amplification and overexpression are significantly correlated in mucinous epithelial ovarian cancer. Hum Pathol 45: 810-816, 2014

10. Bai H, Li H, Li W, Gui T, Yang J, Cao D and Shen K: The $\mathrm{PI} 3 \mathrm{~K} / \mathrm{AKT} / \mathrm{mTOR}$ pathway is a potential predictor of distinct invasive and migratory capacities in human ovarian cancer cell lines. Oncotarget 6: 25520-25532, 2015.

11. Burkhalter RJ, Symowicz J, Hudson LG, Gottardi CJ and Stack MS: Integrin regulation of beta-catenin signaling in ovarian carcinoma. J Biol Chem 286: 23467-23475, 2011.

12. Takayama Y, Kamimura Y, Okawa M, Muramatsu S, Sugino A and Araki H: GINS, a novel multiprotein complex required for chromosomal DNA replication in budding yeast. Genes Dev 17: $1153-1165,2003$

13. Chang YP, Wang G, Bermudez V, Hurwitz J and Chen XS Crystal structure of the GINS complex and functional insights into its role in DNA replication. Proc Natl Acad Sci USA 104: 12685-12690, 2007.

14. MacNeill SA: Structure and function of the GINS complex, a key component of the eukaryotic replisome. Biochem J 425: 489-500, 2010.

15. Kang YH, Galal WC, Farina A, Tappin I and Hurwitz J: Properties of the human Cdc45/Mcm2-7/GINS helicase complex and its action with DNA polymerase epsilon in rolling circle DNA synthesis. Proc Natl Acad Sci USA 109: 6042-6047, 2012.

16. Tumini E, Plevani P, Muzi-Falconi M and Marini F: Physical and functional crosstalk between Fanconi anemia core components and the GINS replication complex. DNA Repair (Amst) 10: 149-158, 2011.

17. Barkley LR, Song I, Zou Y and Vaziri C: Reduced expression of GINS complex members induces hallmarks of pre-malignancy in primary untransformed human cells. Cell Cycle 8: 1577-1588, 2009.
18. Thomassen M, Tan Q and Kruse TA: Gene expression meta-analysis identifies chromosomal regions and candidate genes involved in breast cancer metastasis. Breast Cancer Res Treat 113: 239-249, 2009

19. Rantala JK, Edgren H and Lehtinen L: Integrative functional genomics analysis of sustained polyploidy phenotypes in breast cancer cells identifies an oncogenic profile for GINS2 Neoplasia 12: 877-888, 2010

20. Zheng M, Zhou Y, Yang X, Tang J, Wei D, Zhang Y, Jiang JL, Chen ZN and Zhu P: High GINS2 transcript level predicts poor prognosis and correlates with high histological grade and endocrine therapy resistance through mammary cancer stem cells in breast cancer patients. Breast Cancer Res Treat 148: 423-436, 2014.

21. Peng L, Song Z, Chen D, Linghu R, Wang Y, Zhang X, Kou X, Yang $J$ and Jiao S: GINS2 regulates matrix metallopeptidase 9 expression and cancer stem cell property in human triple negative breast cancer. Biomed Pharmacother 84: 1568-1574, 2016.

22. Liu M, Pan H, Zhang F, Zhang Y, Zhang Y, Xia H, Zhu J, Fu W and Zhang X: Identification of TNM stage-specific genes in lung adenocarcinoma by genome-wide expression profiling. Oncol Lett 6: 763-768, 2013.

23. Gao Y, Wang S, Liu B and Zhong L: Roles of GINS2 in K562 human chronic myelogenous leukemia and NB4 acute promyelocytic leukemia cells. Int J Mol Med 31: 1402-1410, 2013.

24. Zhang X, Zhong L, Liu BZ, Gao YJ, Gao YM and Hu XX: Effect of GINS2 on proliferation and apoptosis in leukemic cell line. Int J Med Sci 10: 1795-1804, 2013

25. Livak KJ and Schmittgen TD: Analysis of relative gene expression data using real-time quantitative PCR and the 2(-delta delta (T))method. Methods 25: 402-408, 2001.

26. Bertoli C, Skotheim JM and de Bruin RA: Control of cell cycle transcription during G1 and S phases. Nat Rev Mol Cell Biol 14: 518-528, 2013.

27. Fisher RP: CDKs and cyclins in transition(s). Curr Opin Genet Dev 7: 32-38, 1997.

28. Yabuuchi H, Yamada Y, Uchida T, Sunathvanichkul T, Nakagawa T and Masukata H: Ordered assembly of Sld3, GINS and Cdc45 is distinctly regulated by DDK and CDK for activation of replication origins. EMBO J 25: 4663-4674, 2006.

29. Choi JM, Lim HS, Kim JJ, Song OK and Cho Y: Crystal structure of the human GINS complex. Genes Dev 21: 1316-1321, 2007.

30. Cohen SM, Chastain PD II, Cordeiro-Stone M and Kaufman DG: DNA replication and the GINS complex: Localization on extended chromatin fibers. Epigenetics Chromatin 2: 6, 2009.

31. Tessema M, Lehmann U and Kreipe H: Cell cycle and no end. Virchows Arch 444: 313-323, 2004. 